\title{
Sex Differences in the Relation Between Subjective Memory Complaints, Impairments in Instrumental Activities of Daily Living, and Risk of Dementia
}

\author{
Herrer Abdulrahman ${ }^{\mathrm{a}, \mathrm{b}, *}$, Edo Richard ${ }^{\mathrm{b}, \mathrm{c}}$, Willem A. van Gool ${ }^{\mathrm{a}, \mathrm{c}}$, Eric P. Moll van Charante ${ }^{\mathrm{a}, \mathrm{c}}$ \\ and Jan Willem van Dalen ${ }^{\mathrm{a}, \mathrm{c}}$ \\ a Amsterdam University Medical Center, University of Amsterdam, Department of Neurology, Amsterdam, \\ the Netherlands \\ ${ }^{\mathrm{b}}$ Radboud University Medical Center, Donders Institute for Brain, Cognition and Behavior, Department \\ of Neurology, Nijmegen, the Netherlands \\ ${ }^{\mathrm{c}}$ Amsterdam University Medical Center, University of Amsterdam, Department of Public and Occupational \\ Health, Amsterdam, the Netherlands
}

Accepted 11 October 2021

Pre-press 15 November 2021

\begin{abstract}
.
Background: Older people with subjective memory complaints (SMC) and Instrumental Activities of Daily Living impairments (IADL-I) have an increased risk of developing dementia. Previous reports suggest that the predictive value of SMC and IADL-I may differ between sexes, leaving possible consequences for personalized risk prediction and prognosis. However, none of these studies addressed the competing risk of death, which may substantially differ between sexes.

Objective: We investigated sex-differences in the association between IADL-I, SMC, and incident dementia and mortality as competing risk.

Methods: 3,409 community-dwelling older people without dementia (mean age 74.3 \pm 2.5 ), were followed for 6.7 years (median). Baseline SMC were assessed using the 15-item Geriatric Depression Scale memory question, and IADL-I using the Academic Medical Center Linear Disability Score. Potential sex-differences in the predictive value of SMC and IADL-I were assessed using Cox regression models with an interaction term for sex.

Results: HRs for isolated SMC and SMC + IADL-I and risk of dementia were higher in women (HR: 2.02, 95\% CI $=0.91-4.46$, $p=0.08 ; \mathrm{HR}: 2.85,95 \% \mathrm{CI}=1.65-4.91, p<0.001)$ than in men (HR:1.52, 95\% CI $=0.86-2.69, p=0.18 ; \mathrm{HR}: 1.24,95 \%$ $\mathrm{CI}=0.62-2.49, p=0.54)$, but these sex-differences were not significant. Conversely, HRs for isolated IADL-I and risk of mortality were higher in men (HR:1.56, 95\% CI $=1.18-2.05, p=0.002)$ than in women $(\mathrm{HR}: 1.14,95 \% \mathrm{CI}=0.80-1.62$, $p=0.48$ ), but again, these sex-differences were not significant.

Conclusion: The predictive value of SMC and IADL-I for the risk of dementia and mortality was not significantly modified by sex. However, the competing risk of death for these factors differed considerably between men and women, suggesting it is an essential factor to consider when comparing sex-differences in IADL/dementia risk.
\end{abstract}

Keywords: Alzheimer's disease, dementia, Instrumental Activities of Daily Living, sex, subjective memory complaints

\footnotetext{
*Correspondence to: Herrer Abdulrahman, MD, Radboud University Medical Center, Department of Neurology, Reinier
} 


\section{INTRODUCTION}

Subjective memory complaints (SMC), operationalized as the subjective awareness of memory loss without deficits on objective testing, affect approximately $25 \%$ of people aged 60 years and older, with higher prevalence with higher age [1-3]. People of 60 years and older with SMC have a two to four times higher risk of developing dementia within five years of reporting SMC [4-6].

SMC can present with impairments in Instrumental Activities of Daily Living (IADL-I), those activities that allow an individual to live independently $[7,8]$. IADL-I are often reported as risk factors for incidentdementia in combination with SMC [9-11]. SMC and IADL-I are common in the aging population, with reports suggesting a prevalence of up to $50 \%$ for both symptoms in community-dwelling older people [12, 13].

Two studies suggest that the relation between SMC, IADL-I, and risk of dementia, may differ between men and women, with SMC conveying increased risk of incident dementia in women, but not in men [14, 15]. Conversely, IADL-I have been reported to be associated with in an increased risk for dementia in men, but not in women [15]. Hypothetically, these findings can be explained by sex-differences in the perception, evaluation, and action towards health problems [16]. Allegedly, women are more self-conscious and therefore more likely to perceive changes in their health [17].

However, these studies did not take the potential competing risk of mortality into account, which may substantially impact the outcomes, since sexdifferences in survival may also influence dementia risk. Understanding sex-differences within this context, could be useful for a more personalized risk prediction and follow-up strategy in clinical practice.

We investigated whether sex modified the predictive value of SMC and IADL-I as risk factors for incident all-cause dementia and evaluated the influence of the competing risk of death, in a large cohort of community-dwelling older people with virtually complete follow-up for dementia and mortality.

\section{MATERIALS AND METHODS}

\section{Study population}

Our study population consisted of communitydwelling older people aged 70-78 years at baseline who participated in the Prevention of Dementia by Intensive Vascular care (preDIVA) trial. This was a multicenter, cluster-randomized controlled trial, evaluating the effectiveness of practice nurseled cardiovascular risk management compared to usual care by the general practitioner for the prevention of dementia [18]. In a population-based approach, community-dwelling older people (aged 70-78 years) registered with one of the participating general practices ( $>98 \%$ of the Dutch general population is registered with a GP) were invited to participate in the trial. Only individuals with dementia or conditions likely to hinder successful long-term follow-up (e.g., terminal illness and alcoholism) were excluded at baseline. Because the preDIVA intervention did not affect the risk of dementia, we studied the study population as a single cohort for our current research question $[18,19]$. The study was approved by the medical ethics committee of the Academic Medical Center Amsterdam, Netherlands. Participants gave written informed consent prior to their baseline visit.

\section{Clinical assessment and cognitive screening}

Participants were assessed at baseline, and at subsequent 2-yearly assessments over 6-8 years of follow-up. At these assessments, data on demographic characteristics, living status, cardiovascular risk factors, cognitive status, (instrumental) activities of daily living, symptoms of depression, and medication use were collected and cross-checked with the participants' electronic health records. To screen for cognitive impairment, at baseline and every 2 years thereafter, cognitive status was evaluated using the Mini-Mental State Examination (MMSE) to assess global cognitive functioning, and the Visual Association Test (VAT), a test that is sensitive to detect impairment of anterograde episodic memory $[19,20]$.

Individuals with an MMSE score $<24$ at baseline were evaluated by their general practitioner and excluded from the study if they might have (possible) dementia. At the follow-up visits, all available clinical information referring to a clinical diagnosis of (possible) incident dementia since the last study visit was gathered. Individuals with low or declining MMSE scores at cognitive screening during follow-up $(<24$ points or $>2$ points decline compared to the previous 2-yearly assessment) were referred to their general practitioner (GP) for clinical evaluation, followed by referral to a memory clinic, at the discretion of the GP, to establish or exclude a diagnosis of incident dementia. 


\section{Dementia diagnosis}

Incident dementia was based on a clinical diagnosis according to DSM-IV criteria during the 6-8 years follow-up, confirmed by an independent, blinded outcome adjudication committee, based on all available clinical information [21]. This committee consisted of neurologists, old-age psychiatrists, geriatricians, cardiologists, and general practitioners. Dementia diagnoses were re-evaluated after one year of additional follow-up to avoid false-positive diagnoses. IADL-impairment and SMC scores obtained at the study assessments were not considered in the dementia diagnostic procedure. For participants who dropped out of the study prematurely, all available information on dementia status was retrieved at the end of the study by a research nurse from electronic health records and contact with the general practitioner, and presented to the outcome adjudication committee.

\section{SMC and IADL-I}

SMC at baseline were operationalized for this study using item 10 of the 15 -item Geriatric Depression Scale (GDS), 'Do you feel you have more problems with memory than most?' [22]. This item has regularly been used by previous studies to measure SMC [23].

ADL were assessed using the Academic Medical Center Linear Disability Score (ALDS) [24]. The ALDS uses a generic, non-disease-specific item bank, consisting of 77 ADL items ordered from simple to complex. ALDS-scores were linearly transformed, resulting in a linear scale with scores ranging from 10-90. For the current analysis, ALDS-scores were dichotomized below and above the median score.

For the analyses, participants were divided into four mutually exclusive groups: isolated SMC (individuals with SMC without IADL-I), isolated IADL-I (individuals with IADL-I without SMC), SMC + IADL-I (individuals with both IADL-I and SMC), and individuals with neither symptom.

\section{Statistical analyses}

We compared baseline characteristics between men and women using Fisher's exact tests for proportions, Wilcoxon signed rank tests for non-normally distributed variables, and Student's $T$-tests for normally distributed variables. For the primary analyses, hazard ratios (HRs) for dementia were calculated using Cox regression, with age as timescale and age at baseline as time of entry. Proportional hazard assumptions were assessed by visually inspecting Schoenfeld residuals and Goodness of Fit tests.

We evaluated the HRs for individuals with isolated SMC, isolated IADL-I, and SMC + IADL-I, compared to individuals with neither SMC nor IADL-I as the reference group, using a categorical variable dividing participants into these four mutually exclusive groups.

Model 1 adjusted for sex, model 2 additionally adjusted for education (categorized as $<7$ years, 7-12 years, and $>12$ years), living status (single/not single), antihypertensive use (yes/no), history of stroke, heart disease, diabetes, and total scores on the GDS, MMSE, and VAT. Living status was added as a confounder because it may influence both health behavior, and whether or not symptoms are noticed and reported [25]. MMSE and VAT were included to adjust for slight measurable differences in cognitive functioning that may not constitute 'objectifiable cognitive impairment' but may differentiate individuals with SMC from those without. The GDS was included because depressive symptoms are associated with subjective memory complaints and also, independently, with an increased risk of dementia [26-28]. History of stroke, heart disease, diabetes, and antihypertensive use were all included as factors that often differ between men and women and may influence IADL, SMC, and dementia risk [29-31]. Individuals were left out per model if any covariates were missing (i.e., pairwise).

Because the competing risk of death may be a major etiological factor in the relationship between cognitive and/or IADL symptoms and dementia, specifically when assessing sex-differences, we assessed the influence of the competing risk of death, using a cause-specific hazard approach: repeating all analyses with mortality and mortality/dementia combined as outcomes $[32,33]$. This approach was favored over a subdistribution hazard approach (e.g., Fine-Gray analysis), because we were interested in the etiological relations between IADL, SMC mortality, and dementia. For this, the cause-specific approach is the most appropriate, because it allows for evaluation of the extent to which a decrease in dementia risk associated with a predictor might be due to an increase in mortality [34].

To assess sex-differences in HRs, risks for dementia, mortality, and dementia-mortality combined were first assessed in the total population, and subsequently in subgroups for men and women separately. 


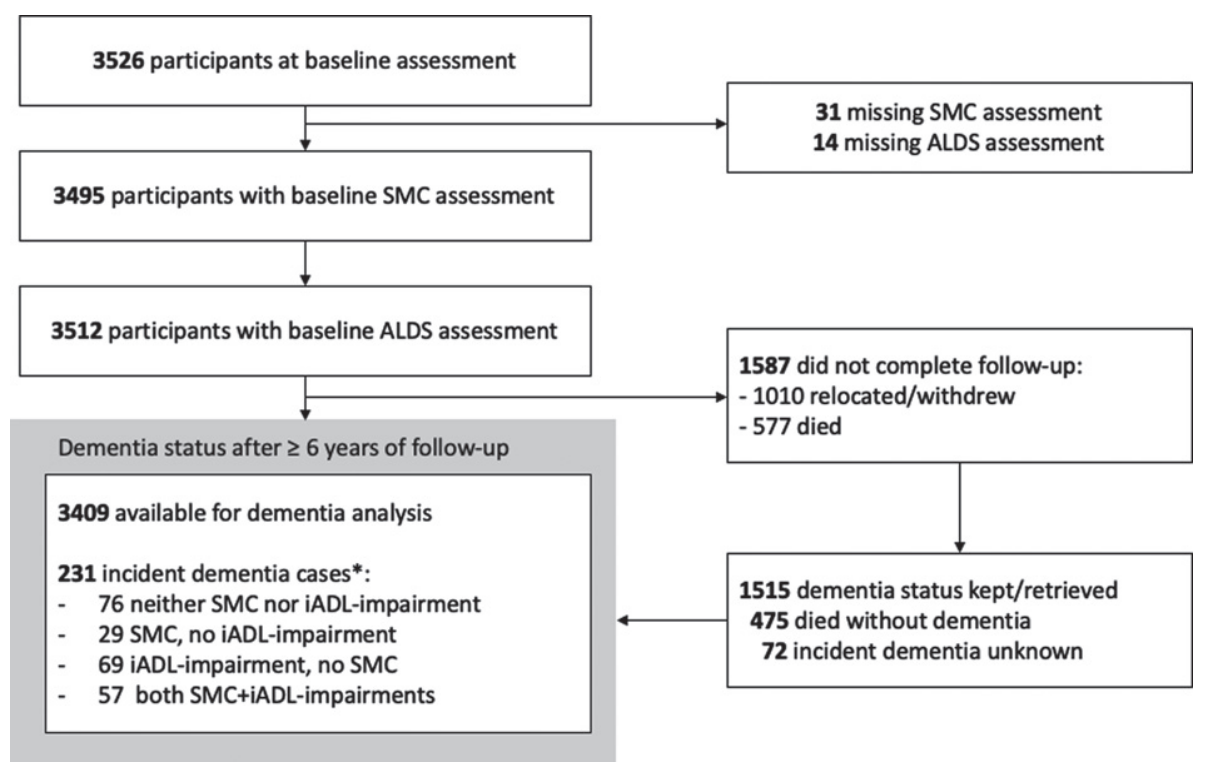

Fig. 1. Study flow-chart * Dementia cases with data available on status of SMC and IADL-Impairment.

To assess whether the association between SMC/IADL-I and each outcome status differed significantly between men and women, we used $p$-values from interaction terms with sex for the individual SMC/IADL categories using dummy variables in the overall population (i.e., isolated SMC (yes/no) * sex; isolated IADL (yes/no)-I* sex; and SMC+IADL-I (yes/no) * sex). Furthermore, we tested the differences between models including and not including these interaction terms with likelihood-ratio tests to assess the $p$-value for the overall interaction of sex with SMC/IADL-I groups.

\section{Sensitivity analyses}

We performed several sensitivity analyses. First, because median ALDS scores may differ between men and women, a sensitivity analysis was performed basing the definition of IADL-I on the median ALDS scores for men and women separately [15]. Second, to compare our results with previous work, we performed a sensitivity analysis with IALD-I defined based on six items relating more specifically to cognitive tasks selected based on face validity [15]. Third, because the diagnosis of SMC formally excludes individuals with mild cognitive impairment (MCI), we evaluated the influence of baseline cognitive status on our findings. We did not search for participants with MCI in our extensive cognitive screening at baseline, therefore an additional sensitivity analysis was performed comparing participants with a base- line MMSE-score of $\geq 28$, with participants with a lower baseline MMSE-score of $<28$. Analyses were performed using R 3.6.1.

\section{RESULTS}

Of 3,526 participants attending preDIVA baseline, $31(0.9 \%)$ were excluded due to missing SMC status and $14(0.4 \%)$ participants were excluded due to missing ALDS-scores. Follow-up for all-cause dementia was complete in $97.9 \%$ of participants, resulting in 3,409 participants $(21,764$ person-years) that were included in the main analyses (Fig. 1). These participants did not differ in baseline characteristics from those excluded, except for more often being single.

Baseline characteristics for the population included are in Table 1 . The mean age was 74.4 $( \pm 2.5)$ years, $54.4 \%$ were women and $45.6 \%$ men. Men and women differed significantly in dementia risk factors. Women more often had low education $(29.4 \%$ versus $18.0 \%, p<0.001)$ and more often lived alone $(45.7 \%$ versus $18.2, p<0.001)$. Men more often had a history of heart disease $(37.3 \%$ versus $23.0 \%, p<0.001)$ and stroke $(11.4 \%$ versus $8.6 \%, p=0.005)$.

In total, $17.6 \%(601 / 3,409)$ of participants reported SMC at baseline. SMC were significantly $(p<0.001)$ less common in women $(15.1 \%, 284 / 1,890)$ than men $(20.6 \%, 327 / 1,591)$. The median ALDS score was $89.2(\mathrm{IQR}=86.4-89.5)$. The median ALDS-score 
Table 1

Population characteristics at baseline of the whole population, and men and women separately

\begin{tabular}{|c|c|c|c|c|}
\hline & $\begin{array}{c}\text { Total } \\
(n=3,409)\end{array}$ & $\begin{array}{c}\text { Men } \\
(n=1,553)\end{array}$ & $\begin{array}{c}\text { Women } \\
(n=1,856)\end{array}$ & $p^{*}$ \\
\hline Age in years, mean $( \pm \mathrm{SD})$ & $74.4(2.5)$ & $74.3(2.5)$ & $74.4(2.4)$ & 0.41 \\
\hline Living alone, $n(\%)$ & $949(33.1)$ & $239(18.2)$ & $710(45.7)$ & $<0.001$ \\
\hline \multicolumn{5}{|l|}{ Education level } \\
\hline$<7$ years, $n(\%)$ & $817(24.2)$ & $276(18.0)$ & $541(29.4)$ & \multirow[t]{3}{*}{$<0.001$} \\
\hline $7-12$ years, $n(\%)$ & $1,915(56.7)$ & $829(54.1)$ & $1086(58.9)$ & \\
\hline$>12$ years, $n(\%)$ & $643(19.1)$ & $427(27.9)$ & $216(11.7)$ & \\
\hline \multicolumn{5}{|l|}{ Cardiovascular risk factors } \\
\hline History of heart disease (IHD), $n(\%)$ & $999(29.5)$ & $575(37.3)$ & $424(23.0)$ & $<0.001$ \\
\hline History of stroke, $n(\%)$ & $333(9.9)$ & $176(11.4)$ & $157(8.6)$ & 0.005 \\
\hline History of Diabetes mellitus, $n(\%)$ & $623(18.3)$ & $302(19.4)$ & $321(17.3)$ & 0.11 \\
\hline Antihypertensive drugs, $n(\%)$ & $1,747(51.5)$ & $796(51.9)$ & $951(51.8)$ & 0.19 \\
\hline \multicolumn{5}{|l|}{ Cognitive assessments } \\
\hline MMSE-score, median (IQR) & $28(27-29)$ & $28(27-29)$ & $29(28-29)$ & 0.38 \\
\hline VAT, median (IQR) & $6(-6)$ & $6(5-6)$ & $6(5-6)$ & 0.42 \\
\hline \multicolumn{5}{|l|}{ Activities of daily living } \\
\hline ALDS-score, median (IQR) & $89.2(86.4-89.5)$ & $89.5(88.1-89.5)$ & $88.1(85.5-89.5)$ & 0.70 \\
\hline \multicolumn{5}{|l|}{ Depression and SMC } \\
\hline GDS-15 score, median (IQR) & $0(0-1)$ & $0(0-1)$ & $0(0-1)$ & 0.98 \\
\hline \multicolumn{5}{|l|}{ SMC/IADL-status: } \\
\hline Neither SMC nor IADL-I, $n(\%)$ & $1,362(40.0)$ & $781(50.3)$ & $581(31.3)$ & $<0.001$ \\
\hline Isolated SMC, $n(\%)$ & $250(7.3)$ & $182(11.7)$ & $68(3.7)$ & $<0.001$ \\
\hline Isolated IADL-II, $n(\%)$ & $1,446(42.4)$ & $452(29.1)$ & $994(53.6)$ & $<0.001$ \\
\hline SMC+IADL-I, $n(\%)$ & $351(10.3)$ & $138(8.9)$ & $213(11.5)$ & 0.02 \\
\hline
\end{tabular}

MMSE, Mini-Mental State Examination; VAT, Visual Association Test; ALDS, Academic Medical Center Linear Disability Score; GDS-sum score, 15-item Geriatric Depression Scale score at baseline; SMC, Subjective Memory Complaints; IADL-I, IADL-Impairments. SMC/IADL status categorized in 4 mutually exclusive groups as used in the analyses: Isolated SMC: individuals with SMC without IADL-I; Isolated IADL-I: individuals with IADL-I without SMC; SMC + IADL-I: individuals with both symptoms. Missings (men/women): Living alone: 243/302, Education: 21/13, History of heart disease: 10/13, History of stroke: 10/25, Antihypertensive drugs: 19/19, MMSE-score: 4/2, VAT: $11 / 6 .{ }^{*} p$-value for difference between men and women.

for women (88.1, IQR = 85.5-89.5) was lower than for men $(89.5, \mathrm{IQR}=88.1-89.5)$, but this difference was not significant $(p=0.70)$. Of the 3,409 participants in total, $7.3 \%$ had isolated SMC $(250 / 3,409)$, $42.4 \%$ had isolated IADL-I $(1,446 / 3,409), 10.3 \%$ had SMC + IADL-I $(351 / 3,409)$, and the remaining $40.0 \%$ had neither symptom $(1,362 / 3,409)$.

After a median of 6.7 years (IQR 6.0-7.2) of follow-up (21,764 person-years), $6.8 \%(231 / 3,409)$ of participants developed dementia and $16.1 \%$ $(549 / 3,409)$ died. Dementia incidence in the four SMC/IADL groups was: $11.6 \%$ in isolated SMC (29/250), $4.8 \%$ in isolated IADL-I $(69 / 1,446), 16.2 \%$ in SMC+IADL-I (57/351), and 5.6\% in the neither symptom group $(76 / 1,362)$. Kaplan-Meier curves for incident dementia in women and men are depicted in Fig. 2.

\section{Dementia risk}

Table 2A lists the results for the main analyses. Results reported in the text below are from the fully adjusted model 2 .
Individuals with isolated SMC, had an $85 \%$ higher dementia risk $(\mathrm{HR}=1.85,95 \% \mathrm{CI}=1.17-2.90$, $p=0.008)$ compared to those with neither symptom. The HR for women with isolated SMC versus women with neither symptom was slightly higher $(2.02,95 \% \mathrm{CI}=0.91-4.46, p=0.08)$ than for men with isolated SMC versus men with neither symptom $(1.52,95 \% \mathrm{CI}=0.86-2.69, p=0.15)$. This difference in HRs for men and women was not significant ( $p$ interaction $=0.57$ ).

Individuals with isolated IADL-I had a nonsignificant $30 \%$ lower risk of dementia $(\mathrm{HR}=0.70$, 95\% CI $=0.48-1.00, p=0.053$ ), compared to those with neither symptom. This HR was slightly higher in women $(\mathrm{HR}=0.77,95 \% \mathrm{CI}=0.48-1.25, p=0.29)$ than in men $(\mathrm{HR}=0.66,95 \% \mathrm{CI}=0.36-1.20$, $p=0.17$ ), but this difference in HRs was not significant $(p$-interaction $=0.67)$.

Individuals with SMC+IADL-I had double the dementia risk $(\mathrm{HR}=2.03,95 \% \mathrm{CI}=1.34-3.06, p=$ $0.001)$ compared to individuals with neither symptom. The HR was higher in women $(\mathrm{HR}=2.85,95 \%$ $\mathrm{CI}=1.65-4.91, p<0.001)$ than in men $(\mathrm{HR}=$ 


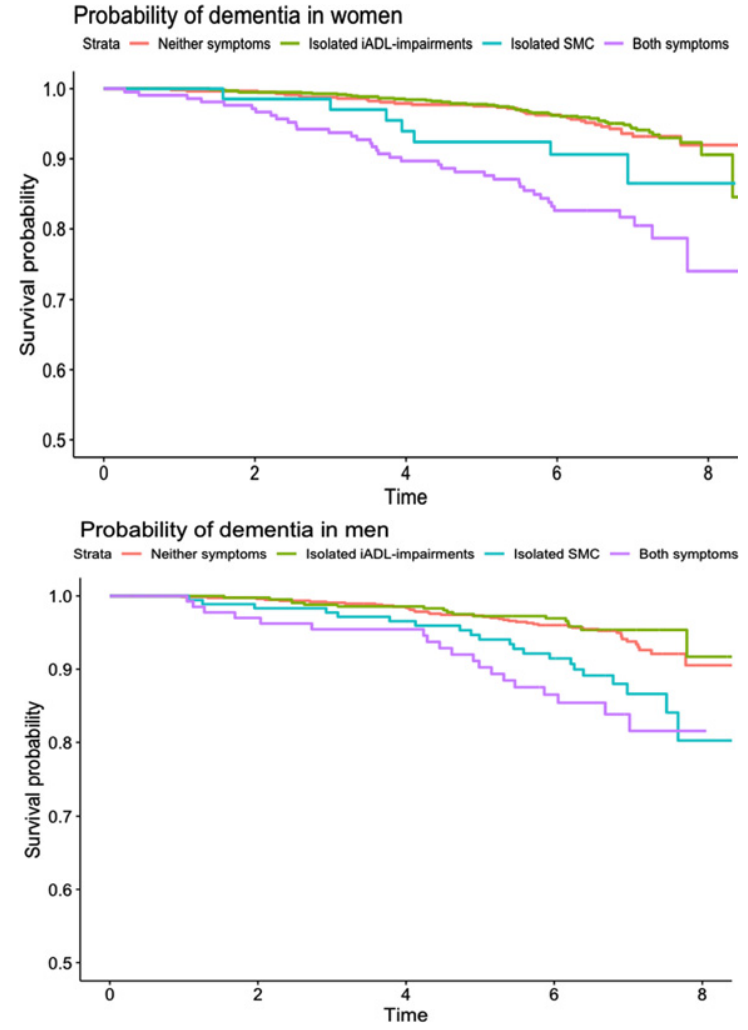

Fig. 2. Kaplan-Meier curves for probability of dementia in men and women over 8 years of study follow-up.
$1.24,95 \% \mathrm{CI}=0.62-2.49, p=0.54)$, but this difference in HRs was not significant ( $p$-interaction $=0.06$ ).

The overall difference between the models for dementia with and without interaction terms for sex with the individual SMC/IADL categories was also non-significant ( $p$-value overall interaction: $0.47)$.

\section{Competing risk of death}

To evaluate the competing risk of death, Table $2 \mathrm{~B}$ and Supplementary Figure 1 present the results of analyses with mortality as outcome.

Individuals with isolated SMC had a nonsignificant $20 \%$ higher risk of mortality $(\mathrm{HR}=1.20$, 95\% CI $=0.85-1.69, p=0.29)$ compared to those with neither symptom. This HR was slightly higher for women with isolated SMC versus women with neither symptom $(\mathrm{HR}=1.36,95 \% \mathrm{CI}-0.70-2.66$, $p=0.36$ ) than for men with isolated SMC versus men with neither symptom $(\mathrm{HR}=1.21,95 \%$ $\mathrm{CI}=0.81-1.80, p=0.36)$, but this difference in HRs was not significant $(p$-interaction $=0.76)$.

Individuals with isolated IADL-I had a $30 \%$ greater risk of mortality $(\mathrm{HR}=1.36,95 \% \mathrm{CI}=1.09-1.68$, $p=0.006)$ compared to those with neither symptom. This HR was lower in women $(\mathrm{HR}=1.14,95 \%$ $\mathrm{CI}=0.80-1.62, p=0.48)$ than in men $(\mathrm{HR}=1.56$,

Table 2A

Risk of dementia in individuals with isolated SMC, isolated impairments in IADL, or both (SMC + IADL-I), compared to individuals with neither (No SMC/IADL-I), in the total population and stratified according to sex

\begin{tabular}{|c|c|c|c|c|c|c|c|c|}
\hline \multirow[b]{2}{*}{ Total: } & \multicolumn{4}{|c|}{$\begin{array}{c}\text { Model } 1 \\
n \text { events }=231, \mathrm{n} \text { total }=3,409\end{array}$} & \multicolumn{4}{|c|}{$\begin{array}{c}\text { Model } 2 \\
n \text { events }=214, \mathrm{n} \text { total }=3,263\end{array}$} \\
\hline & events/tot & HR & $95 \% \mathrm{CI}$ & $p$ & events/tot & HR & $95 \% \mathrm{CI}$ & $p$ \\
\hline No SMC/IADL-I & $76 / 1362$ & 1.00 & (reference) & & $72 / 1312$ & 1.00 & (reference) & \\
\hline Isolated SMC & $29 / 250$ & 2.15 & $1.40-3.31$ & $<0.001$ & $27 / 236$ & 1.85 & $1.17-2.90$ & 0.008 \\
\hline Isolated IADL-I & $69 / 1446$ & 0.80 & $0.57-1.12$ & 0.19 & $64 / 1383$ & 0.70 & $0.48-1.00$ & 0.053 \\
\hline SMC + IADL-I & $57 / 351$ & 3.04 & $2.14-4.30$ & $<0.001$ & $51 / 332$ & 2.03 & $1.34-3.06$ & 0.001 \\
\hline Men:* & \multicolumn{4}{|c|}{$n$ events $=100, n$ total $=1553$} & \multicolumn{4}{|c|}{$n$ events $=90, n$ total $=1479$} \\
\hline No SMC/IADL-I & $43 / 781$ & 1.00 & (reference) & & $40 / 753$ & 1.00 & (reference) & \\
\hline Isolated SMC & $21 / 182$ & 2.17 & $1.29-3.65$ & $<0.01$ & $19 / 170$ & 1.52 & $0.86-2.69$ & 0.15 \\
\hline Isolated IADL-I & $17 / 452$ & 0.70 & $0.40-1.24$ & 0.22 & $17 / 427$ & 0.66 & $0.36-1.20$ & 0.17 \\
\hline SMC + IADL-I & $19 / 138$ & 2.73 & $1.59-4.71$ & $<0.001$ & $14 / 129$ & 1.24 & $0.62-2.49$ & 0.54 \\
\hline Women: ${ }^{*}$ & \multicolumn{4}{|c|}{$n$ events $=131, n$ total $=1856$} & \multicolumn{4}{|c|}{$n$ events $=124, n$ total $=1784$} \\
\hline No SMC/IADL-I & $33 / 581$ & 1.00 & (reference) & & $32 / 559$ & 1.00 & (reference) & \\
\hline Isolated SMC & $8 / 68$ & 2.09 & $0.96-4.53$ & 0.06 & $8 / 66$ & 2.02 & $0.91-4.46$ & 0.08 \\
\hline Isolated IADL-I & $52 / 994$ & 0.87 & $0.56-1.35$ & 0.54 & $47 / 956$ & 0.77 & $0.48-1.25$ & 0.29 \\
\hline SMC + IADL-I & $38 / 213$ & 3.34 & $2.09-5.35$ & $<0.001$ & $37 / 203$ & 2.85 & $1.65-4.91$ & $<0.001$ \\
\hline
\end{tabular}

Hazard ratio's (HR) and 95\% confidence intervals (95\% CI) from Cox regression analyses accounting for age and sex (model 1), additionally adjusted for education, living-status, history of stroke, heart disease, diabetes, antihypertensive use, and mini-mental examination, geriatric depression scale, and visual association test scores (model 2). ${ }^{*} p$-values for interactions for men versus women, for each IADL/SMC group compared to the reference category: Model 1: Isolated SMC* ${ }^{*} \operatorname{sex}, p=0.94$; Isolated IADL-I* $\operatorname{sex}, p=0.56$; SMC +IADL-I* sex $p=0.21$. Model 2: Isolated SMC*sex, $p=0.57$; Isolated IADL-I*sex, $p=0.67$; SMC + IADL-I* $\operatorname{sex} p=0.06 .{ }^{*} p$-values for the overall interaction (models including vs not including sex interaction terms): Model 1: $p=0.93$, Model 2: $p=0.47$. 
Table 2B

Mortality risk in individuals with isolated SMC, isolated impairments in IADL, or both (SCM + IADL-I), compared to individuals with neither (No SMC/IADL-I), in the total population and stratified according to sex

\begin{tabular}{|c|c|c|c|c|c|c|c|c|}
\hline \multirow[b]{2}{*}{ Total: } & \multicolumn{4}{|c|}{$\begin{array}{c}\text { Model } 1 \\
n \text { events }=549, n \text { total }=3,405\end{array}$} & \multicolumn{4}{|c|}{$\begin{array}{c}\text { Model } 2 \\
n \text { events }=526, n \text { total }=3,259\end{array}$} \\
\hline & events/tot & HR & $95 \% \mathrm{CI}$ & $p$ & events/tot & HR & $95 \% \mathrm{CI}$ & $p$ \\
\hline No SMC/IADL-I & $163 / 1360$ & 1.00 & (reference) & & $160 / 1310$ & 1.00 & (reference) & \\
\hline Isolated SMC & $44 / 250$ & 1.33 & $0.95-1.86$ & 0.09 & $43 / 236$ & 1.20 & $0.85-1.69$ & 0.29 \\
\hline Isolated IADL-I & $260 / 1444$ & 1.76 & $1.44-2.16$ & $<0.001$ & $244 / 1381$ & 1.36 & $1.09-1.68$ & 0.006 \\
\hline SMC + IADL-I & $82 / 351$ & 2.20 & $1.68-2.88$ & $<0.001$ & $79 / 332$ & 1.43 & $1.06-1.95$ & 0.02 \\
\hline Men: ${ }^{*}$ & \multicolumn{4}{|c|}{$n$ events $=316, n$ total $=1,551$} & \multicolumn{4}{|c|}{$n$ events $=300, n$ total $=1,477$} \\
\hline No SMC/IADL-I & $115 / 780$ & 1.00 & (reference) & & $113 / 752$ & 1.00 & (reference) & \\
\hline Isolated SMC & $33 / 182$ & 1.22 & $0.83-1.81$ & 0.30 & $32 / 170$ & 1.21 & $0.81-1.80$ & 0.36 \\
\hline Isolated IADL-I & $125 / 451$ & 1.93 & $1.50-2.50$ & $<0.001$ & $114 / 426$ & 1.56 & $1.18-2.05$ & 0.002 \\
\hline SMC+ IADL-I & $43 / 138$ & 2.21 & $1.55-3.14$ & $<0.001$ & $41 / 129$ & 1.59 & $1.07-2.37$ & 0.02 \\
\hline Women: ${ }^{*}$ & \multicolumn{4}{|c|}{$n$ events $=131, n$ total $=1,856$} & \multicolumn{4}{|c|}{$n$ events $=226, n$ total $=1,782$} \\
\hline No SMC/IADL-I & $48 / 580$ & 1.00 & (reference) & & $47 / 558$ & 1.00 & (reference) & \\
\hline Isolated SMC & $11 / 68$ & 1.87 & $0.97-3.60$ & 0.06 & $11 / 66$ & 1.36 & $0.70-2.66$ & 0.36 \\
\hline Isolated IADL-I & $135 / 993$ & 1.56 & $1.12-2.17$ & $<0.001$ & $130 / 955$ & 1.14 & $0.80-1.62$ & 0.48 \\
\hline SMC+ IADL-I & $39 / 213$ & 2.12 & $1.39-3.24$ & $<0.001$ & $38 / 203$ & 1.22 & $0.76-1.97$ & 0.41 \\
\hline
\end{tabular}

Hazard ratio's (HR) and 95\% confidence intervals (95\% CI) from Cox regression analyses accounting for age and sex (model 1), additionally adjusted for education, living-status, history of stroke, heart disease, diabetes, antihypertensive use, and mini-mental examination, geriatric depression scale, and visual association test scores (model 2). ${ }^{*} p$-values for interactions for men versus women, for each IADL/SMC group compared to the reference category: IADL-I. Model 1: Isolated SMC*sex, $p=0.29$; Isolated IADL-I* sex, $p=0.31$; SMC+IADL$I^{*} \operatorname{sex} p=0.87$. Model 2: Isolated SMC* ${ }^{*} \operatorname{sex}, p=0.76$; Isolated IADL-I* $\operatorname{sex}, p=0.16$; SMC +IADL-I* $\operatorname{sex} p=0.38$. ${ }^{*} p$-values for the overall interaction (models including vs not including sex interaction terms): Model 1: $p=0.37$, Model 2: $p=0.54$.

95\% CI $=1.18-2.05, p=0.002)$, but this difference in HRs was not significant ( $p$-interaction $=0.16)$.

Individuals with SMC + IADL-I had a higher risk of mortality compared to those with neither symptom $(\mathrm{HR}=1.43,95 \% \mathrm{CI}=1.06-1.95, p=0.02)$. This $\mathrm{HR}$ was slightly lower in women $(\mathrm{HR}=1.22,95 \%$ $\mathrm{CI}=0.76-1.97, p=0.41)$ than in men $(\mathrm{HR}=1.59$, $95 \% \mathrm{CI}=1.07-2.37, p=0.02)$, but this difference in HRs was not significant ( $p$-interaction $=0.38$ ).

The overall difference between the models for mortality with and without interaction terms for sex with the individual SMC/IADL categories was also non-significant ( $p$-value overall interaction: 0.54 ).

With dementia/mortality as combined outcome (Supplementary Figure 2 and Supplementary Table 1), HRs were greater than 1 for all categories, suggesting that overall SMC and IADL convey an increased risk of poor dementia/mortality outcome, and that in individuals with isolated IADL-I, the decreased risk of incident dementia was outweighed by the increased risk of mortality. There were no significant differences in HRs for dementia/mortality between sexes.

\section{Sensitivity analyses}

When IADL-I were determined using separate cut-off ALDS-scores for men and women based on the median ALDS score for each (Supplementary Table 2A), HRs for individuals with isolated IADL-I compared those having neither symptom were similar for men $(\mathrm{HR}=0.83,95 \% \mathrm{CI}=0.48-1.45)$ and women $(\mathrm{HR}=0.77,95 \% \mathrm{CI}=0.48-1.23)$. The mortality-risk in those with isolated IADL-I was similar to previous models (Supplementary Table 2B).

When basing IADL-I on items with an important cognitive component (Supplementary Table 3A), individuals with isolated IADL-I had a nonsignificant $20 \%$ lower dementia risk than those with neither symptom $(\mathrm{HR}=0.7995 \% \mathrm{CI}=0.45-1.39$, $p=0.41$ ). This HR was particularly low (albeit nonsignificant $)$ in men ( $\mathrm{HR}=0.40,95 \% \mathrm{CI}=0.13-1.22$, $p=0.11$ ), while the HR was neutral in women (HR $=1.09,95 \% \mathrm{CI}=0.56-2.14)$. These riskdifferences between men and women were not significant $(p=0.13)$.

Similarly to the main analyses, individuals with isolated IADL-I had a greater HR for mortality compared to individuals with neither symptom. This HR was greater in women $(\mathrm{HR}=1.89,95 \%$ $\mathrm{CI}=1.30-2.75, p<0.001)$ than in men $(\mathrm{HR}=1.11$, $95 \% \mathrm{CI}=0.73-1.70, p=0.62$ ), but these differences in risk-effect were not significant (Supplementary Table 3B).

Lastly, sensitivity analysis including participants with possible MCI (Supplementary Table 4A, B) showed similar results to the main analysis. 


\section{DISCUSSION}

In our cohort of 3,409 community-dwelling older people, individuals with isolated IADL-I had a $30 \%$ lower, those with isolated SMC an $85 \%$ higher, and those with both IADL-I and SMC an 103\% higher risk of dementia, compared to those with neither symptom, during 6-8 years of follow-up. HRs for SMC with dementia were generally higher in women than in men but these sex-differences were not significant. The dementia risk decrease associated with isolated IADL-I was generally greater in men, but again the sex-differences in HRs were not significant. Individuals with isolated IADL-I had a higher risk of mortality and dementia/mortality combined, suggesting that the lower dementia risk associated with isolated IADL-I could be related to the competing risk of death. Associations with mortality were stronger in men, suggesting that competing risk of death is an essential factor to consider when comparing sexdifferences in dementia risk. Basing IADL-I only on IADL-I tasks pertaining to cognition also gave similar results. Basing IADL-I on sex-specific median scores negated the sex-differences in predictive value.

Overall, the nearly double risk of incident dementia associated with isolated SMC in our study, is comparable to findings in similar community-dwelling older populations [6]. Only two studies have reported investigating sex-differences in the context of SMC and dementia risk in community-dwelling older people, both finding that SMC increased dementia risk in women $(+77 \%$ and $+88 \%)$ but not in men $(-1 \%$ and $+7 \%)[14,15]$. In our study, the HRs for dementia associated with SMC also seemed higher in subgroups of women than in subgroups of men, but the sex-differences in association did not reach statistical significance. In addition, although not significant when fully adjusted, HRs for SMC categories in men in our study did suggest higher dementia risk $(+24$ and $+52 \%)$. Our analyses adjusted for more confounders, but this is unlikely to have caused the differential findings, since our sensitivity analysis matching the confounders in the previous studies gave largely similar results. An explanation may be that SMC were stronger predictors of dementia in our study, with an overall HR of 1.88 compared to approximately 1.50 in the previous studies. Possibly, this reflects the different operationalization of SMC: our study inquired whether participants experienced more memory complaints than most (of similar age), the other studies documented whether individuals experienced memory symptoms in general. In addition, our study population was generally younger, healthier, and higher educated $[14,15]$. Perception of memory problems in healthier, higher educated, relatively young older people may more often be related to brain disease instead of normal aging and could therefore be better predictors of dementia $[16,35,36]$. Supporting this explanation, the prevalence of SMC was much higher in the previous studies $(62 \%$ and $58 \%$ ) compared to ours (18\%), and thereby potentially more often related to normal ageing instead of developing cognitive decline. It should be considered that the lower baseline age of our population is likely to contribute a to the lower percentage of SMC compared to previous studies. Hypothetically, sex-differences in the predictive value of SMC are caused by sex-differences in perception, evaluation, and action towards health problems, with women being more attentive to health [17]. Because the association with dementia was stronger in our population compared to the previous studies', the predictive power of SMC in men may have been less attenuated.

Our finding that isolated IADL-I increase mortality risk follow the growing body of evidence that IADL-I are independent risk-factors for mortality in older people [37-39]. Whether isolated IADL-I independently predict incident dementia has been little evaluated, since they are generally only considered as additional risk-factor in the context of more severe cognitive decline (e.g., MCI) $[9,11,40]$. One study found that isolated IADL-I double the risk of dementia in community-dwelling older people, but in men only [15]. We found that differences in competing risk of mortality may be important, but whether these might explain the differential study results cannot be ascertained since the previous study did not investigate mortality risks. However, the lower dementia risk in our study cannot be explained by increased mortality rates since fewer people died in our study. Another explanation could be that we used the ALDS to assess IADL-I, which comprises both cognitive and physical items. However, our results remained similar when we based IADL-I on cognitive items only. We found that sex-differences in predictive power of IADL-I may be due to the different distribution of IADL scores in men and women. The median IADL score was somewhat higher in men, and when using sex-specific median cut-off values to define IADL-I, the predictive value of IADL-I was similar in men and women. Previous reports show that women tend to score lower on IADL questionnaires, and cut-off scores to define substantial IADL-I should therefore 
be tailored to sex-differences [41, 42]. Hypothetically, women are more aware and more inclined to report health (changes), partly due to societal views on sex-differences in caregiving roles and acknowledgement of physical discomfort [42, 43]. Women have been found to be more attentive towards declining IADL, and more likely to underestimate their abilities, while men more often overestimate their abilities, leading to lower IADL scores for women compared to men $[42,44]$.

Taken together, our results suggest that lower dementia risks associated with isolated IADL-I are likely due to increased risk of mortality, and that IADL-I only increase dementia risk when in the context of SMC.

Our findings that individuals with SMC+IADLI gave the highest risk of incident dementia supports the use of IADL-I as additional risk factor (or perhaps early symptom), increasing the risk of developing dementia when observed in the context of cognitive symptoms. However, the additional value of IADL-I in combination with SMC in the general population setting seems relatively limited, conveying only marginally increased dementia risk compared to isolated symptoms, while the additional value is reportedly much greater in the context of more severe cognitive symptoms like MCI [42, 44]. Potentially, this is because individuals generally develop severe IADL-I in more advanced stages of cognitive decline, and earlier IADL-I are often caused by other age related, non-cognitive, impairments.

This study has limitations. First, the absence of significant differences between sexes for some analyses is likely due to some of the subgroups being relatively small, leading to low statistical power for the fully adjusted model. This is particularly illustrated in the main analysis (Table 2) in which the fully adjusted HR for dementia was much higher in women with SMC + IADL-I than in men, but this difference was not significant $(p=0.06)$. This is likely due to subgroup of men with SMC+IADL-I having too few dementia cases for the number of covariates in model 2. However, although the risk conveyed by SMC for dementia may be higher in women, it remains that in our study, SMC consistently conveyed increased dementia risks in both sexes. Second, because SMC were derived from screening in a general population of community-dwelling older people, results may not directly apply to spontaneously expressed SMC and/or the memory clinic population. Third, in our baseline diagnostic cognitive screening assessment, we excluded participants with dementia, but we did not formally identify those with objectifiable memory complaints. Therefore, we cannot state with complete certainty that our study population did not include participants with objectifiable memory complaints. The likelihood of our results being driven by participants with objectifiable cognitive impairment is considered low, because the sensitivity analysis in which we compare lower MMSE- $(<28)$ with higher MMSE-score $(\geq 28)$ participants, yielded similar HRs as the main analysis. In addition, we adjusted for MMSE and VAT-scores in all our analyses, thereby maximally correcting for such a potential effect.

Fourth, we adjusted for cognitive functioning using the MMSE, a relatively crude cognitive measure, and the VAT, which has high specificity but low sensitivity for predicting dementia [45]. These could have missed very subtle cognitive deficits. However, this would likely affect both sexes similarly and previous studies evaluating sex-differences adjusted for cognitive functioning only using the MMSE-score, so this cannot likely explain the different study results. Fifth, because we used a crude measure to assess SMC ("'Do you feel you have more problems with memory than most?"), risk-differences associated with SMC severity between men and women, may have been obscured, analogues to our findings in IADLI. Interestingly however, although previous studies in community-dwelling older people found significantly higher prevalences of SMC for both men compared to women and vice versa, the higher predictive value of SMC in women is consistent, suggesting that sex-specific cut-off values for SMC severity may not fully negate the difference $[14,15]$. Sixth, we defined IADL-I based on a uniform set for both sexes but, due to differences in gender roles and expectations, men and women may experience IADL-I in different tasks [46].

Lastly, one must consider that IADL-I is a relative term in this study, because of the ceiling effect on the ALDS-score. We assessed IADL-I using the median ALDS-score in our relatively healthy study population of community dwelling older people. This median ALDS-score, used as a cut-off score for determining IADL-I, was relatively high compared to what one would expect to find in a general older population or in memory clinic patients. Based on findings from previous studies, the strong association between mortality and our definition of IADL-I suggests that the IADL-I cut-off value used in our study is comparable to mild IADL-I [37, 47, 48]. These studies showed similar HRs between IADL-I and risk of 
mortality in community dwelling older people with mild IADL-I. Participants with IADL-I in this study population might therefore represent a milder spectrum of IADL-I.

Strengths of our study are the 6-8-year follow-up including 2-yearly cognitive assessment, and the virtually complete data for dementia outcome $(98 \%)$. Therefore, incident dementia was unlikely to remain undetected throughout the study, and the risk of (selective) attrition influencing study results was minimal. Furthermore, clinical dementia diagnoses were validated by an independent committee of medical specialists and general practitioners. As a quality check, dementia diagnoses were re-evaluated after one year, minimizing the risk of false-positive diagnoses. In addition, we reported on the risk of mortality in the context of SMC, IADL, and dementia, showing that mortality may strongly influence dementia risks, and should be taken into account in future studies, especially considering that older women have a substantially lower mortality risk despite higher frailty [49]. Lastly, several sensitivity analyses were performed to better understand the role of IADLI, suggesting that differences in the distribution of IADL-I between men and women, might account for the differences in the predictive value. This highlights the potential importance of using sex-standardized cut-off scores when predicting dementia risk both in the clinical and in the research setting.

\section{Conclusion}

In our large cohort of community-dwelling older people, individuals with isolated SMC and individuals with both SMC and IADL-I had a significantly greater risk of developing dementia, whereas individuals with isolated IADL-I did not. The HR of SMC without IADL-I was higher in women compared to the HR in men, but this difference was not significant. However, the competing risk of death for these factors differed considerably between men and women, suggesting it is an essential factor to consider when comparing sex-differences in IADL/dementia risk. These effects could become even more apparent in cohorts with higher mortality rates. Although the present findings do not warrant strong sex-specific recommendations with respect to risk prediction, usage of sex-specific cut-off values for IADL-I on self-report questionnaires might be relevant considerations for researchers and health professionals investigating IADL-I.

\section{ACKNOWLEDGMENTS}

This project is funded by The Netherlands Organization for Health Research and Development (ZonMw) VIDI grant 91718303 to E. Richard. The funder did not play a role in any part (such as initiation, execution, or interpretation of the results) of this manuscript. The corresponding author affirms that she has listed everyone who contributed significantly to the work. All authors meet the criteria for authorship stated in the Uniform Requirements for Manuscripts Submitted to Biomedical Journals.

Authors' disclosures available online (https:// www.j-alz.com/manuscript-disclosures/21-5191r2).

\section{SUPPLEMENTARY MATERIAL}

The supplementary material is available in the electronic version of this article: http://dx.doi.org/ 10.3233/JAD-215191.

\section{REFERENCES}

[1] Montejo P, Montenegro M, Fernandez MA, Maestu F (2011) Subjective memory complaints in the elderly: Prevalence and influence of temporal orientation, depression and quality of life in a population-based study in the city of Madrid. Aging Ment Health 15, 85-96.

[2] Jonker C, Geerlings MI, Schmand B (2000) Are memory complaints predictive for dementia? A review of clinical and population-based studies. Int J Geriatr Psychiatry 15, 983-991.

[3] Abdulrab K, Heun R (2008) Subjective Memory Impairment. A review of its definitions indicates the need for a comprehensive set of standardised and validated criteria. Eur Psychiatry 23, 321-330.

[4] Mendonça MD, Alves L, Bugalho P (2016) From subjective cognitive complaints to dementia: Who is at risk?: A systematic review. Am J Alzheimers Dis Other Demen 31, 105-114.

[5] Tobiansky R, Blizard R, Livingston G, Mann A (1995) The Gospel Oak Study stage IV: The clinical relevance of subjective memory impairment in older people. Psychol Med 25, 779-786.

[6] Mitchell AJ, Beaumont H, Ferguson D, Yadegarfar M, Stubbs B (2014) Risk of dementia and mild cognitive impairment in older people with subjective memory complaints: Meta-analysis. Acta Psychiatr Scand 130, 439-451.

[7] Ryu SY, Lee SB, Kim TW, Lee TJ (2016) Subjective memory complaints, depressive symptoms and instrumental activities of daily living in mild cognitive impairment. Int Psychogeriatr 28, 487-494.

[8] Rotenberg Shpigelman S, Sternberg S, Maeir A (2019) Beyond memory problems: Multiple obstacles to health and quality of life in older people seeking help for subjective memory complaints. Disabil Rehabil 41, 19-25.

[9] Barberger-Gateau P, Fabrigoule C, Helmer C, Rouch I, Dartigues JF (1999) Functional impairment in instrumental 
activities of daily living: An early clinical sign of dementia? $J$ Am Geriatr Soc 47, 456-462.

[10] Cloutier S, Chertkow H, Kergoat MJ, Gélinas I, Gauthier S, Belleville S (2021) Trajectories of decline on instrumental activities of daily living prior to dementia in persons with mild cognitive impairment. Int J Geriatr Psychiatry 36, 314323.

[11] Yam A, Marsiske M (2013) Cognitive longitudinal predictors of older adults' self-reported IADL function. J Aging Health 25, 163s-185s.

[12] Wolfsgruber S, Kleineidam L, Wagner M, Mösch E, Bickel H, Lühmann D, Ernst A, Wiese B, Steinmann S, König HH, Brettschneider C, Luck T, Stein J, Weyerer S, Werle J, Pentzek M, Fuchs A, Maier W, Scherer M, Riedel-Heller SG, Jessen F (2016) Differential risk of incident Alzheimer's disease dementia in stable versus unstable patterns of subjective cognitive decline. J Alzheimers Dis 54, 1135-1146.

[13] Ponds RW, Commissaris KJ, Jolles J (1997) Prevalence and covariates of subjective forgetfulness in a normal population in The Netherlands. Int J Aging Hum Dev 45, 207-221.

[14] Heser K, Kleineidam L, Wiese B, Oey A, Roehr S, Pabst A, Kaduszkiewicz H, van den Bussche H, Brettschneider C, König HH, Weyerer S, Werle J, Fuchs A, Pentzek M, Mösch E, Bickel H, Maier W, Scherer M, Riedel-Heller SG, Wagner M (2019) Subjective cognitive decline may be a stronger predictor of incident dementia in women than in men. J Alzheimers Dis 68, 1469-1478.

[15] Pérès K, Helmer C, Amieva H, Matharan F, Carcaillon L, Jacqmin-Gadda H, Auriacombe S, Orgogozo JM, Barberger-Gateau P, Dartigues JF (2011) Gender differences in the prodromal signs of dementia: Memory complaint and IADL-restriction. a prospective populationbased cohort. J Alzheimers Dis 27, 39-47.

[16] Thompson AE, Anisimowicz Y, Miedema B, Hogg W, Wodchis WP, Aubrey-Bassler K (2016) The influence of gender and other patient characteristics on health care-seeking behaviour: A QUALICOPC study. BMC Fam Pract 17, 38.

[17] Verbrugge LM (1982) Sex differentials in health. Public Health Rep 97, 417-437.

[18] Moll van Charante EP, Richard E, Eurelings LS, van Dalen JW, Ligthart SA, van Bussel EF, Hoevenaar-Blom MP, Vermeulen M, van Gool WA (2016) Effectiveness of a 6-year multidomain vascular care intervention to prevent dementia (preDIVA): A cluster-randomised controlled trial. Lancet 388, 797-805.

[19] Folstein MF, Folstein SE, McHugh PR (1975) “Mini-mental state". A practical method for grading the cognitive state of patients for the clinician. J Psychiatr Res 12, 189-198.

[20] Lindeboom J, Schmand B, Tulner L, Walstra G, Jonker C (2002) Visual association test to detect early dementia of the Alzheimer type. J Neurol Neurosurg Psychiatry 73, 126133.

[21] Bell CC (1994) DSM-IV: Diagnostic and Statistical Manual of Mental Disorders. JAMA 272, 828-829.

[22] Sheikh JI, Yesavage JA (1986) Geriatric Depression Scale (GDS): Recent evidence and development of a shorter version. Clin Gerontol 5, 165-173.

[23] Rabin LA, Smart CM, Crane PK, Amariglio RE, Berman LM, Boada M, Buckley RF, Chételat G, Dubois B, Ellis KA, Gifford KA, Jefferson AL, Jessen F, Katz MJ, Lipton RB, Luck T, Maruff P, Mielke MM, Molinuevo JL, Naeem F, Perrotin A, Petersen RC, Rami L, Reisberg B, Rentz DM, Riedel-Heller SG, Risacher SL, Rodriguez O, Sachdev PS, Saykin AJ, Slavin MJ, Snitz BE, Sperling RA, Tandetnik C, van der Flier WM, Wagner M, Wolfsgruber
S, Sikkes SA (2015) Subjective cognitive decline in older adults: An overview of self-report measures used across 19 international research studies. J Alzheimers Dis 48 Suppl 1, S63-86.

[24] Holman R, Lindeboom R, Glas CAW, Vermeulen M, de Haan RJ (2003) Constructing an item bank using item response theory: The AMC Linear Disability Score project. Health Serv Outcomes Res Methodol 4, 19-33.

[25] Joung IM, Stronks K, van de Mheen H, Mackenbach JP (1995) Health behaviours explain part of the differences in self reported health associated with partner/marital status in The Netherlands. J Epidemiol Community Health 49, 482488.

[26] Singh-Manoux A, Dugravot A, Ankri J, Nabi H, Berr C, Goldberg M, Zins M, Kivimaki M, Elbaz A (2014) Subjective cognitive complaints and mortality: Does the type of complaint matter? J Psychiatr Res 48, 73-78.

[27] da Silva J, Gonçalves-Pereira M, Xavier M, MukaetovaLadinska EB (2013) Affective disorders and risk of developing dementia: Systematic review. Br J Psychiatry 202, 177-186.

[28] Diniz BS, Butters MA, Albert SM, Dew MA, Reynolds CF, 3rd (2013) Late-life depression and risk of vascular dementia and Alzheimer's disease: Systematic review and meta-analysis of community-based cohort studies. Br J Psychiatry 202, 329-335.

[29] Hayden KM, Zandi PP, Lyketsos CG, Khachaturian AS, Bastian LA, Charoonruk G, Tschanz JT, Norton MC, Pieper CF, Munger RG, Breitner JC, Welsh-Bohmer KA (2006) Vascular risk factors for incident Alzheimer disease and vascular dementia: The Cache County study. Alzheimer Dis Assoc Disord 20, 93-100.

[30] Reeves MJ, Bushnell CD, Howard G, Gargano JW, Duncan PW, Lynch G, Khatiwoda A, Lisabeth L (2008) Sex differences in stroke: Epidemiology, clinical presentation, medical care, and outcomes. Lancet Neurol 7, 915-926.

[31] Ljungman C, Kahan T, Schiöler L, Hjerpe P, Hasselström J, Wettermark B, Boström KB, Manhem K (2014) Gender differences in antihypertensive drug treatment: Results from the Swedish Primary Care Cardiovascular Database (SPCCD). J Am Soc Hypertens 8, 882-890.

[32] Harada CN, Natelson Love MC, Triebel KL (2013) Normal cognitive aging. Clin Geriatr Med 29, 737-752.

[33] Noordzij M, Leffondré K, van Stralen KJ, Zoccali C, Dekker FW, Jager KJ (2013) When do we need competing risks methods for survival analysis in nephrology? Nephrol Dial Transplant 28, 2670-2677.

[34] van Dalen JW, Moll van Charante EP, Richard E, van Gool WA (2018) Antihypertensive drugs, incident dementia, and the competing risk of death. J Am Med Dir Assoc 19, 10261027.

[35] Abner EL, Kryscio RJ, Caban-Holt AM, Schmitt FA (2015) Baseline subjective memory complaints associate with increased risk of incident dementia: The PREADVISE trial. J Prev Alzheimers Dis 2, 11-16.

[36] van Oijen M, de Jong FJ, Hofman A, Koudstaal PJ, Breteler MM (2007) Subjective memory complaints, education, and risk of Alzheimer's disease. Alzheimers Dement 3, 92-97.

[37] Hennessy S, Kurichi JE, Pan Q, Streim JE, Bogner HR, Xie D, Stineman MG (2015) Disability stage is an independent risk factor for mortality in Medicare beneficiaries aged 65 years and older. $P M R$ 7, 1215-1225.

[38] Wu LW, Chen WL, Peng TC, Chiang ST, Yang HF, Sun YS, Chan JY, Kao TW (2016) All-cause mortality risk in elderly 
individuals with disabilities: A retrospective observational study. BMJ Open 6, e011164.

[39] Millán-Calenti JC, Tubío J, Pita-Fernández S, GonzálezAbraldes I, Lorenzo T, Fernández-Arruty T, Maseda A (2010) Prevalence of functional disability in activities of daily living (ADL), instrumental activities of daily living (IADL) and associated factors, as predictors of morbidity and mortality. Arch Gerontol Geriatr 50, 306-310.

[40] Merrill SS, Seeman TE, Kasl SV, Berkman LF (1997) Gender differences in the comparison of self-reported disability and performance measures. J Gerontol A Biol Sci Med Sci 52, M19-26.

[41] Boerma T, Hosseinpoor AR, Verdes E, Chatterji S (2016) A global assessment of the gender gap in self-reported health with survey data from 59 countries. BMC Public Health 16, 675.

[42] Botoseneanu A, Allore HG, Mendes de Leon CF, Gahbauer EA, Gill TM (2016) Sex differences in concomitant trajectories of self-reported disability and measured physical capacity in older adults. J Gerontol A Biol Sci Med Sci 71, 1056-1062.

[43] Ikeda Y, Ogawa N, Yoshiura K, Han G, Maruta M, Hotta M, Tabira T (2019) Instrumental Activities of Daily Living: The processes involved in and performance of these activities by Japanese community-dwelling older adults with subjective memory complaints. Int J Environ Res Public Health 16, 2617.
[44] Jekel K, Damian M, Wattmo C, Hausner L, Bullock R, Connelly PJ, Dubois B, Eriksdotter M, Ewers M, Graessel E, Kramberger MG, Law E, Mecocci P, Molinuevo JL, Nygård L, Olde-Rikkert MG, Orgogozo JM, Pasquier F, Peres K, Salmon E, Sikkes SA, Sobow T, Spiegel R, Tsolaki M, Winblad B, Frölich L (2015) Mild cognitive impairment and deficits in instrumental activities of daily living: A systematic review. Alzheimers Res Ther 7, 17.

[45] Jongstra S, van Gool WA, Moll van Charante EP, van Dalen JW, Eurelings LSM, Richard E, Ligthart SA (2018) Improving prediction of dementia in primary care. Ann Fam Med 16, 206-210.

[46] Sheehan CM, Tucker-Drob EM (2019) Gendered expectations distort male-female differences in Instrumental Activities of Daily Living in later adulthood. J Gerontol B Psychol Sci Soc Sci 74, 715-723.

[47] Zhang Z, Xie D, Kurichi JE, Streim J, Zhang G, Stineman MG (2012) Mortality predictive indexes for the communitydwelling elderly US population. J Gen Intern Med 27, 901910.

[48] Stineman MG, Xie D, Pan Q, Kurichi JE, Zhang Z, Saliba D, Henry-Sánchez JT, Streim J (2012) All-cause 1-, 5-, and 10 -year mortality in elderly people according to activities of daily living stage. J Am Geriatr Soc 60, 485-492.

[49] Gordon EH, Peel NM, Samanta M, Theou O, Howlett SE, Hubbard RE (2017) Sex differences in frailty: A systematic review and meta-analysis. Exp Gerontol 89, 30-40. 